\section{Nonadherence to guidelines in the management of hepatocellular carcinoma: an Italian or universal phenomenon?}
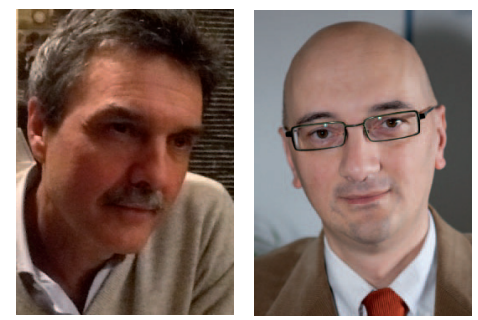

“... by carefully adapting guidelines at
a domestic level we can improve
quality of healthcare and outcomes for

our patients, with knock-on

improvements in survival."

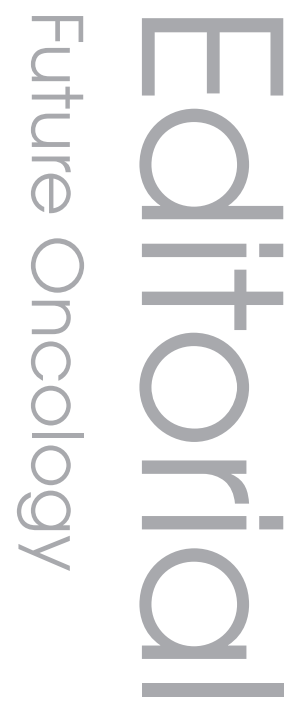

Mauro Borzio*1 \& Rodolfo Sacco 2

Unità di Gastroenterologia, Azienda Ospedaliera di Melegnano, Melegnano, Italy

2Unità di Gastroenterologia, Ospedale Cisanello, Pisa, Italy

*Author for correspondence: Tel.. + 390292360317 - Fax: + 390292360347 - mauro.borzio@gmail.com

According to the definition by Grimshaw and Russell, clinical practice guidelines are "systematically developed statements to assist practitioner and patient decisions about appropriate healthcare for specific clinical circumstances" [1]. Guidelines are specifically devoted to successfully support effective clinical practice and improve quality of care with the aim of obtaining a final health gain. However, application and, consequently, adherence to guidelines is seldom as optimal as expected, and adherence is critical in translating recommendations into improved outcomes.

A variety of possible barriers that undermine adherence have been identified. They have been categorized as physician-related barriers (e.g., lack of awareness, familiarity, agreement, credibility and outcome expectancy), patient-related barriers (e.g., acceptance) and environmentalrelated barriers (e.g., resources or availability and accessibility of facilities). All of these barriers would account for a disappointingly low adherence to guidelines in clinical practice. It is indeed widely accepted that guidelines have a limited effect on changing physician behavior. Therefore, the implementation of measures to improve adherence and the detection of barriers hindering the effectiveness of guidelines is an important goal for the scientific community worldwide.

Over the last two decades, the management of hepatocellular carcinoma (HCC) has witnessed an overall increased complexity due to striking advances in diagnostic techniques and more effective treatment methods, which determine significant increases in survival [2]. This prompted experts to produce and regularly update clinical practice guidelines. In total, 16 academic-drafted guidelines from different geographical areas are now available, the most important and popular of them being the North American and European guidelines, which were first released in 2001 and updated in 2005 and 2011 and endorsed by the American Association for the Study of Liver Diseases (AASLD) and the European Association for the Study of the Liver-European Organisation for Research and Treatment of Cancer (EASL-EORTC) scientific societies; the Asian guidelines endorsed by the Asian-Pacific Association for Study of the Liver; and the Japanese guidelines endorsed by the Japan Society of Hepatology. The proliferation of so many guidelines reflects broad geographic differences in HCC epidemiology, etiology, high-risk patients, health systems and resources, medical technology and clinical impact of HCC in different countries. Differences among the guidelines, which may even be substantial, also reflects differences in the organization of varying health systems in terms of availability and accessibility of resources, reimbursement and different physician expertise.

A paradigmatic example of these differences is the role of contrast-enhanced ultrasound (CEUS) in the diagnostic approach to HCC. In the more recent version of AASLD and EASL-EORTC guidelines CEUS has no longer been included among the dynamic imaging techniques for the noninvasive diagnosis of HCC. There are several reasons underlying this exclusion. Noteworthy, in the USA, CEUS is not approved by US FDA and, in addition, ultrasound examination is usually performed by radiology technicians as in many other European countries. On the other

\section{Keywords}

- AASLD guidelines $=$ field practice w hepatocellular carcinoma $=$ Italy = liver cancer $=$ management - sorafenib

\section{Future Medicine part of}


hand, in Italy, as well as in Japan and in many countries of the Far East, CEUS has a very easy accessibility and has been used for a long time by experienced hepatologists and gastroenterogists who are also directly involved in interventional ultrasound. This may explain why CEUS still maintains its pivotal role as a recommended firstline dynamic imaging technique in guidelines from these countries.

Apart from disagreement among different HCC guidelines, the most pressing problem is to evaluate their adherence and applicability in everyday clinical practice, or, in other words, their effectiveness. Unfortunately, only a few studies addressing this topic have been published to date. In the USA, data from SEER register on HCC management found a disappointingly low adherence to AASLD guidelines with respect to application of ultrasound surveillance, diagnostic work-up and proper allocation to the different therapeutic options [3]. Similar conclusions were also reached in a multicenter cooperative survey on hospitalized HCCs carried out in France [4]. The study from Borzio et al., published in the February issue of Future Oncology, was specifically designed to prospectively investigate the adherence to the 2005 AASLD practice guidelines, which were considered the standard-of-care for HCC management in Italy at the time, by collecting data from newly diagnosed HCC patients in 30 general hospitals over a 2-year period [5]. The main result of this study was that in Italian clinical practice adherence to guidelines with respect to surveillance application, diagnostic approach and therapeutic allocation is alarmingly low, particularly in patients with early-stage HCC [6-8].

66...while much effort is put into the development of international guidelines to improve management of hepatocellular carcinoma, there is still a large gap between projected goals and implementation."

As to the low adherence to the AASLD noninvasive diagnostic algorithm, particularly in patients with small HCCs, in a large proportion of patients studied, diagnosis of $\mathrm{HCC}<2 \mathrm{~cm}$ was based on a single imaging procedure and in some cases only on CEUS. These violations from the diagnostic algorithm recommended by AASLD guidelines were associated with a relatively low use of contrast-enhanced MRI $[9,10]$. The authors outline that in Italy MRI is still hardly accessible outside of specialist centers, with longer waiting times as compared with CT scans and CEUS. In addition, the majority of hepatologists are more confident of this latter technique when they are called to quickly characterize any small nodule detected by standard ultrasonography. In fact, as previously stated, the use of CEUS has recently been recommended by the Italian Association for the Study of the Liver (AISF), since the risk of misdiagnosis of intrahepatic cholangiocarcinoma with this technique is minimal ( 2\%) [101].

The study by Borzio et al. also found difficulties in adherence to the therapeutic allocation algorithm recommended by AASLD, which basically relies on the Barcelona Clinic Liver Cancer (BCLC) staging system [5]. According to the BCLC system, the best treatment should be decided on the basis of the stage of HCC [11]. It is noteworthy that the most relevant deviation from the guidelines were an overall under treatment and patients belonging to a specific stage were offered inappropriate treatments. The authors drew attention to the presence of a large number of elderly patients with relevant extrahepatic comorbidities, probably the most relevant barrier contributing to these deviations.

66...we conclude that nonadherence to international guidelines is widespread."

The reliability of BCLC staging as an allocative therapeutic system in clinical practice might be questioned. The BCLC staging system has been endorsed by AASLD and EASL guidelines for almost 15 years. However, it has only been validated internally and externally as a prognostic stage system; in this setting, its reliability is widely accepted. However, some doubts emerged regarding its competence in deciding the best treatment to be offered. The most noticeable weakness concerns therapeutic decisions on patients belonging to the intermediate-stage HCC. They constitute a heterogeneous population, characterized by varying tumor burdens, liver function and disease etiology. According to the BCLC system, all patients in this group should be treated in the same way. In its recent expert report, the AISF outlined the shortcomings of the BLCL system based on the 'one stage, one treatment' concept [101]. We believe that, in order to function in the Italian scenario, management guidelines need to take into account clinical and organizational factors and the possibility of using sequential/combination therapy if first-line therapy fails. 
Another possible explanation for the under treatment found in the study by Borzio et al. may be an inappropriate use of locoregional therapies - percuataneous ethanol injection, radiofrequency ablation or transarterial chemoembolization - instead of liver resection and transplantation [5]. This may occur in real-life clinical practice because clinicians working in nonreferral centers and, consequently, outside specialized teams often overuse locoregional therapies, according to their specific expertise and local resources even though they are not recommended by the guidelines.

The therapeutic approach to patients in the advanced BCLC stage is another setting where AASLD guidelines were largely violated. Since 2008, sorafenib has been the only drug approved for the treatment of patients with BCLC stage C. In addition, this molecule can be used in intermediate-stage (BCLC stage B) patients according to the strict criteria included in the EASL-EORTC guidelines and AISF recommendations. However, Borzio et al. found a dramatic underuse of this treatment in patients with advanced HCC, mainly due to the scarce propensity of hepatologists to undertake an oncologic targeted therapy in every day clinical practice; this could be attributed, at least in part, to the fact that clinical research is still active in identifying patients who can better respond to targeted therapies (e.g., the search for appropriate biomarkers) [5].

The results of this small-scale study in Italy provide a stark demonstration that while much effort is put into the development of international guidelines to improve management of

HCC, there is still a large gap between projected goals and implementation. However, these findings are not peculiar to Italy, as demonstrated for instance in a recent study by Song et al. [12]. To improve standard-of-care for HCC, countries should establish and implement domestic guidelines, which should take into account local resources in terms of health systems, access to diagnostic procedures and technology - and at the same time promote efforts to assess effectiveness and adherence to guidelines when applied in the 'real-life' scenario.

To reply to our opening question - is nonadherence to guidelines in the management of HCC an Italian or universal phenomenon? - we conclude that nonadherence to international guidelines is widespread. However, we do believe that by carefully adapting guidelines at a domestic level we can improve quality of healthcare and outcomes for our patients, with knock-on improvements in survival.

Financial \& competing interests disclosure
The authors have no relevant affliations or financial
involvement with any organization or entity with a
financial interest in or financial conflict with the sub-
ject matter or materials discussed in the manuscript.
This includes employment, consultancies, honoraria,
stock ownership or options, expert testimony, grants or
patents received or pending, or royalties.
Writing assistance was utilized in the production of
this manuscript. Editorial assistance, in terms of
English revision and technical editing, was provided by
L Giacomelli and was supported by internal funds.

\section{Financial \& competing interests disclosure}

The authors have no relevant affiliations or financial involvement with any organization or entity with a financial interest in or financial conflict with the subject matter or materials discussed in the manuscript. This includes employment, consultancies, honoraria, stock ownership or options, expert testimony, grants or atents received or pending, or royalties. English revision and technical editing, was provided by L Giacomelli and was supported by internal funds.

field-practice multicenter study. Future Oncol. 9(2), 283-294 (2013).

1. Grimshaw JM, Russell IT. Achieving health gain through clinical guidelines II: ensuring guidelines change medical practice. Qual. Health Care 3, 45-52 (1994).

2. Santi V, Buccione D, Di Micoli A et al. The changing scenario of hepatocellular carcinoma over the last two decades in Italy. J. Hepatol. 56, 397-405 (2012).

3. Davila JA, Morgan RO, Richardson PA, Du XL, McGlynn KA, El-Serag HB. Use of surveillance for hepatocellular carcinoma among patients with cirrhosis in the United States. Hepatology 52, 132-141 (2010).

4. Rosa I, Denis J, Renard P et al. A French multicentric longitudinal descriptive study of hepatocellular carcinoma management (the CHANGH cohort): preliminary results. J. Hepatol. 53, S231-S232 (2010).

5. Borzio M, Fornari F, De Sio I et al. Adherence to AASLD guidelines for the management of hepatocellular carcinoma: results of an Italian
6. Bruix J, Sherman M, Llovet JM et al. Clinical management of hepatocellular carcinoma. Conclusions of the Barcelona-2000 EASL conference. European Association for the Study of the Liver. J. Hepatol. 35, 421-430 (2001).

7. Bruix J, Sherman M; Practice Guidelines Committee; American Association for the Study of Liver Diseases. Management of hepatocellular carcinoma. Hepatology 42, 1208-1236 (2005).

8. Bruix J, Sherman M. Management of hepatocellular carcinoma, an update. Hepatology 53, 1020-1022 (2011).

9. European Association For The Study of The Liver, European Organisation For Research and Treatment of Cancer. EASL-EORTC clinical practice guidelines: management of hepatocellular carcinoma. J. Hepatol. 56, 908-943 (2012).
10. Vilana R, Forner A, Bianchi L et al. Intrahepatic peripheral cholangiocarcinoma in cirrhosis patients may display a vascular pattern similar to hepatocellular carcinoma on contrast-enhanced ultrasound. Hepatology 51, 2020-2029 (2010).

11. Llovet JM, Brú C, Bruix J. Prognosis of hepatocellular carcinoma: the BCLC staging classification. Semin. Liver Dis. 19, $329-338$ (1999).

12. Song $P$, Tobe RG, Inagaki Y et al. The management of hepatocellular carcinoma around the world: a comparison of guidelines from 2001 to 2011. Liver Int. 32(7), 1053-1063 (2012).

\section{Website}

101. Italian Association for the Study of Liver. [AISF recommendations for the integrated management of HCC patients (2012). www.webaisf.org/media/16110/ raccomandazioni-aisf-per-hcc.pdf 